\title{
Density functional theory study of phase stability, vibrational and electronic properties of $\mathrm{Mo}_{3} \mathrm{Al}_{2} \mathrm{C}$
}

\author{
D. Reith ${ }^{*}$ C. Blaas-Schenner, and R. Podloucky \\ Department of Physical Chemistry, University of Vienna and Center for \\ Computational Materials Science, Sensengasse 8, A-1090 Vienna, Austria
}

(Dated: October 25, 2018)

\begin{abstract}
Based on density functional theory the noncentrosymmetric superconductor $\mathrm{Mo}_{3} \mathrm{Al}_{2} \mathrm{C}$ in its well established $\beta$-Mn type $\left(\mathrm{P} 4_{1} 32\right)$ crystal structure is investigated. In particular, its thermodynamical and dynamical stabilities are studied by calculating lattice vibrations within the harmonic approximation. It is found that the fully stoichiometric compound is dynamically unstable. However, compounds with carbon vacancies, i.e., $\mathrm{Mo}_{3} \mathrm{Al}_{2} \mathrm{C}_{1-x}$, can be dynamically stabilized for vacancy concentrations $x>0.09$. By means of a simple thermodynamical model we estimate $x \sim 0.13-0.14$ for $\mathrm{Mo}_{3} \mathrm{Al}_{2} \mathrm{C}_{1-x}$ at the experimental preparation temperatures. The influence of the carbon vacancy concentration on the electronic structure is investigated.

PACS numbers: 63.20.D-,63.70.+h,71.15.Mb,71.20.-b,61.72.jd
\end{abstract}

\section{INTRODUCTION}

$\mathrm{Mo}_{3} \mathrm{Al}_{2} \mathrm{C}$ has been already synthesized in the $1960 \mathrm{~s}^{1}$ and subsequently classified as a superconductor ${ }^{2 / 5}$ Recently it has attracted renewed attention,,$[10$ because its cubic $\beta$-Mn type crystal structure does not contain a center of inversion. In such noncentrosymmetric superconductors, as first discussed for $\mathrm{CePt}_{3} \mathrm{Si}^{[11}$ the electron pairing process is described to be be a mixture of spinsinglet and spin-triplet states. $\frac{12 / 13}{}$ The question whether $\mathrm{Mo}_{3} \mathrm{Al}_{2} \mathrm{C}$ can be classified as a conventional or unconventional superconductor remains still unresolved according to very recent investigations. ${ }^{6}-8$ In this work, we will not tackle this issue directly but will provide results of extensive density functional theory (DFT) calculations on the thermodynamical and dynamical stability as well as the electronic structure of this compound as a function of carbon content.

$\mathrm{Mo}_{3} \mathrm{Al}_{2} \mathrm{C}$ crystalizes in the cubic $\beta$-Mn type $\mathrm{P} 4_{1} 32$ structure containing 24 atoms in the unit cell with, namely $12 \mathrm{Mo}, 8 \mathrm{Al}$, and $4 \mathrm{C}$ atoms. The $\mathrm{C}$ atoms are in the center of regular $\mathrm{Mo}_{6}$ octahedrons which are tilted to each other! 16 The comparison of our DFT structural parameters to experimental values ${ }^{6}$ in Table 1 shows both in excellent agreement.

\section{COMPUTATIONAL DETAILS}

The DFT calculations were done using the Vienna $a b$ initio simulation package (VASP) $\underline{1415}$ utilizing the pseudopotential construction according to the projector augmented wave method ${ }^{[16}$ For the exchange-correlation functional the generalized gradient approximation as parametrized by Perdew, Burke, and Ernzerho 17 was chosen. The potential for Mo contained 9 valence states including the $4 \mathrm{p}$ semicore states, whereas for $\mathrm{Al}$ and $\mathrm{C}$ three and four valence states were included, respectively. The size of the basis set was defined by an energy cutoff of $500 \mathrm{eV}$. The Brillouin-zone integration for the com-
TABLE I: Structural parameters and Wyckoff positions of $\mathrm{Mo}_{3} \mathrm{Al}_{2} \mathrm{C}$.

\begin{tabular}{|c|c|c|}
\hline & exp. $^{a}$ & $\mathrm{DFT}^{b}$ \\
\hline lattice parameter $c$ & $6.863 \AA$ & $6.890 \AA$ \\
\hline Mo on $12 d$ & $0.2025(2)$ & 0.20183 \\
\hline $\mathrm{Al}$ on $8 \mathrm{c}$ & $0.068(1)$ & 0.06658 \\
\hline \multicolumn{3}{|l|}{$\mathrm{C}$ on $4 \mathrm{a}$} \\
\hline \multirow{2}{*}{$\begin{array}{l}\text { crystal structure: } \\
\text { space group: }\end{array}$} & \multirow{2}{*}{\multicolumn{2}{|c|}{ cubic $\beta$-Mn type }} \\
\hline & & \\
\hline
\end{tabular}

${ }^{a}$ experimental results from Ref. 6]

${ }^{b}$ DFT calculation (present work)

putation of total energies was made using the tetrahedron method with with Blöchl's corrections $\frac{18}{18}$ based on a $13 \times 13 \times 13$ Monkhorst and Pack ${ }^{19} \vec{k}$-point mesh, whereas for the structural relaxations and for the derivation of the force constants the first order Methfessel-Paxton smearing method ${ }^{20}$ on a $7 \times 7 \times 7 \vec{k}$-point mesh was chosen.

The vibrational properties were calculated within the harmonic approximation by making use of the direct force-constant method as implemented in our program package fPHON (full-symmetry PHON), which is based on the package PHON ${ }^{21}$ The structural parameters, i.e., the volume and shape of the unit cell as well as the positions of the atoms within the unit cell, were relaxed until the residual forces were less than $10^{-4} \mathrm{eV} / \AA$. Furthermore, for a high accuracy of the phonon calculations the force constants derived from the displaced atoms were corrected by subtracting the tiny but still finite forces of the fully relaxed structures. Some of the phonon dispersions were cross checked by using density functional perturbation theory ${ }^{222}$ (DFPT) as implemented in VASP. 


\section{VIBRATIONAL PROPERTIES}

It turns out that perfectly stoichiometric $\mathrm{Mo}_{3} \mathrm{Al}_{2} \mathrm{C}$ is dynamically unstable. According to panel (a) of Fig. 1 optical modes with imaginary frequencies around $\Gamma$ arise. The dynamical instability seems surprising considering the well-established crystal structure of $\mathrm{Mo}_{3} \mathrm{Al}_{2} \mathrm{C}$ !10 One should, however, be aware that the actual carbon content is experimentally difficult to discern by X-ray diffraction because of carbons comparatively small Xray cross section $\sqrt{23}$ and therefore there exists some uncertainty with respect to carbon vacancies. In general, many carbides are prone to have vacancies on the $\mathrm{C}$ sublattice.

Before speculating on the physical explanation of the detected instability we undertook numerical and methodological tests. First of all, it is assured that the results are converged with respect to the number of $\vec{k}$ points. Furthermore, for deriving the force constants several atomic displacements of the atoms, i.e., $|\vec{u}|=$ $\{0.01,0.02,0.05,0.1,0.2,0.3,0.4\} \AA$ were chosen and calculations without any symmetry were performed. All calculations confirm the existence of imaginary modes for $\mathrm{Mo}_{3} \mathrm{Al}_{2} \mathrm{C}$. In particular, the calculation with the smallest displacement of $|\vec{u}|=0.01 \AA$ yielded imaginary optical modes with a frequency of $1.62 i \mathrm{THz}$ at $\Gamma$. As a further test the DFPT ${ }^{22}$ technique as implemented in VASP was applied also resulting in imaginary modes of $1.65 i \mathrm{THz}$ at $\Gamma$. From these DFPT calculations force constants have been derived and used as input for $f$ PHON. Both, the direct force-constant method and the DFPT treatment gave very similar results for the phonon dispersions as shown in panels (a) of Fig. 1 .

In order to study if another similar structure exists that is dynamically stable and energetically more favorable compared to the cubic $\beta$-Mn type structure, all atoms were displaced from their equilibrium positions. In addition, a tetragonal deformation was enforced onto the unit cell. Using VASP this deformed structure was subsequently relaxed without any symmetry constrains, resulting in the well-known crystal structure described before.

Because the perfectly stoichiometric compound $\mathrm{Mo}_{3} \mathrm{Al}_{2} \mathrm{C}$ is found to be dynamically unstable, we investigated if dynamical stabilization can be achieved by vacancies, in particular by carbon vacancies.

In general, vacancies on all sublattices will stabilize the phonon dispersion, at least above a certain concentration. Vacancies on the Mo or Al sublattice are less likely to exist since they would be easily detectable, i.e., they have a comparatively large X-ray cross section.23 Furthermore, our DFT derived vacancy formation energies in the following Section IV] strongly indicate that Mo or $\mathrm{Al}$ vacancies are thermodynamically too costly to be formed.

Assuming a certain carbon vacancy concentration suitable supercell calculations were done for the defect structures. Panel (b) in Fig. 1 shows the phonon dispersion for
$\mathrm{Mo}_{3} \mathrm{Al}_{2} \mathrm{C}_{0.75}$, i.e., for a single carbon vacancy in the standard unit cell $(1 \times 1 \times 1$ supercell $)$ with 3 out of possible 4 carbon sites occupied. Further calculations, of which the dispersions are not shown, were also done for a single carbon vacancy in a $2 \times 1 \times 1$ supercell $\left(\mathrm{Mo}_{3} \mathrm{Al}_{2} \mathrm{C}_{0.875}\right.$ with 7 out of possible $8 \mathrm{C}$ sites occupied) and in a $2 \times 2 \times 2$ supercell $\left(\mathrm{Mo}_{3} \mathrm{Al}_{2} \mathrm{C}_{0.96875}\right.$ with 31 out of $32 \mathrm{C}$ sites occupied). Both $\mathrm{Mo}_{3} \mathrm{Al}_{2} \mathrm{C}_{0.75}$ and $\mathrm{Mo}_{3} \mathrm{Al}_{2} \mathrm{C}_{0.875}$ are found to be dynamically stable with no imaginary modes, whereas $\mathrm{Mo}_{3} \mathrm{Al}_{2} \mathrm{C}_{0.96875}$ is found to be significantly unstable with the value of the lowest imaginary optical modes at $\Gamma$ being $1.26 i \mathrm{THz}$ quite close to that of the perfect stoichiometric compound (1.62 $i \mathrm{THz})$.

In Fig. 2 the normalized phonon density of states (DOS) of the dynamically unstable compounds $\mathrm{Mo}_{3} \mathrm{Al}_{2} \mathrm{C}_{1}$ and $\mathrm{Mo}_{3} \mathrm{Al}_{2} \mathrm{C}_{0.96875}$ are compared to those of the dynamically stable compounds $\mathrm{Mo}_{3} \mathrm{Al}_{2} \mathrm{C}_{0.875}$ and $\mathrm{Mo}_{3} \mathrm{Al}_{2} \mathrm{C}_{0.75}$. While at low frequencies no Debye-like $\omega^{2}$ behavior is observed for $\mathrm{Mo}_{3} \mathrm{Al}_{2} \mathrm{C}_{1}$ and $\mathrm{Mo}_{3} \mathrm{Al}_{2} \mathrm{C}_{0.96875}$, it is seen for the other two cases. For $\mathrm{Mo}_{3} \mathrm{Al}_{2} \mathrm{C}_{0.875}$ the Debye-like behavior is observed only in a rather narrow frequency range up to $0.5 \mathrm{THz}$ due to the softening of the acoustical and optical modes. However, for $\mathrm{Mo}_{3} \mathrm{Al}_{2} \mathrm{C}_{0.75}$ the Debye-feature reaches up to $1.4 \mathrm{THz}$. The partial DOS reveals that Mo, as the heaviest atomic species, dominates the lower frequency spectrum (up to 7.5 THz), whereas $\mathrm{C}$, being the lightest atom, has contributions only at frequencies above $13.5 \mathrm{THz}$. Furthermore, the carbon dominated frequency modes are shifted down by the introduction of vacancies from above $15 \mathrm{THz}$ for $\mathrm{Mo}_{3} \mathrm{Al}_{2} \mathrm{C}_{1}$ to about $13.5 \mathrm{THz}$ for $\mathrm{Mo}_{3} \mathrm{Al}_{2} \mathrm{C}_{0.75}$. Strikingly, in all the DOS's a pronounced $\mathrm{Al}$ peak at $\approx$ 12.2 $\mathrm{THz}$ occurs. The Al spectrum is rather broad with significant contributions from $6 \mathrm{THz}$ to $12.2 \mathrm{THz}$. Even below this range a telling small contribution is found indicating a hybridization with Mo modes.

The results indicate, that there is a critical concentration of carbon vacancies below which the compound becomes dynamically unstable. Assuming that the frequency of the lowest optical mode at $\Gamma$ scales linearly with the carbon vacancy concentration $x$, the critical carbon vacancy concentration is estimated to be $x_{\text {crit }} \sim 0.09$ (using the values of $\mathrm{Mo}_{3} \mathrm{Al}_{2} \mathrm{C}_{1-x}$ at $x=0,0.03125$, and 0.125 as input; see Fig. 3 .

The cause of the stabilization of the optical lowfrequency Mo modes by the carbon vacancies is the changed Mo- $\mathrm{C}$ bonding in the $\mathrm{Mo}_{6} \mathrm{C}$ subunits. We examined the relaxations occurring in $\mathrm{Mo}_{3} \mathrm{Al}_{2} \mathrm{C}_{0.75}$, i.e., when a single $\mathrm{C}$ atom is removed from one of the four $\mathrm{Mo}_{6} \mathrm{C}$ subunits in the unit cell of $\mathrm{Mo}_{3} \mathrm{Al}_{2} \mathrm{C}_{1}$. Thereby a strong influence on the Mo- $\mathrm{C}$ bonds in all three remaining $\mathrm{Mo}_{6} \mathrm{C}$ subunits is observed because they share a common Mo atom with the defect subunit. By relaxing the atomic positions the $\mathrm{C}$ atom drifts into an off-center position within the remaining $\mathrm{Mo}_{6} \mathrm{C}$ subunits, increasing the average Mo-C bond length by $1.4 \%$ and the corresponding octahedral volume by $3.6 \%$. The distortion of the remaining $\mathrm{Mo}_{6} \mathrm{C}$ subunits seems to be the stabi- 
(a)

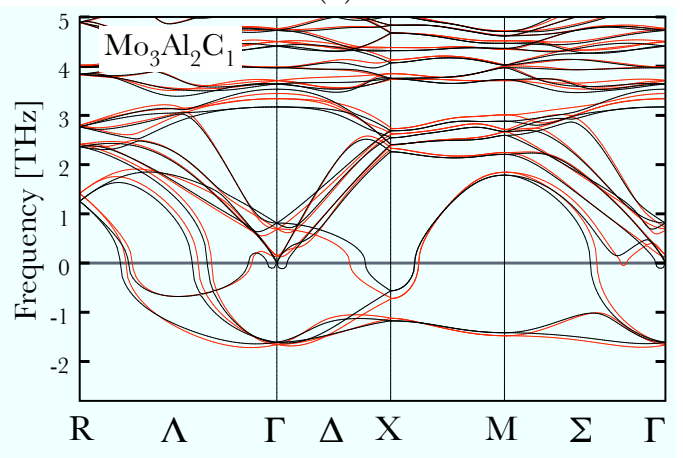

(b)

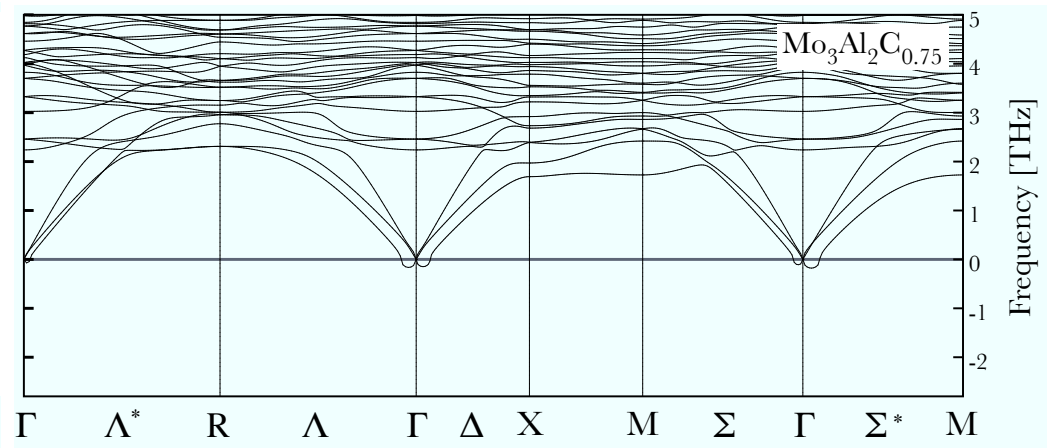

FIG. 1: (Color online) Phonon dispersions up to $5 \mathrm{THz}$ of stoichiometric $\mathrm{Mo}_{3} \mathrm{Al}_{2} \mathrm{C}$ (a) and of $\mathrm{Mo}_{3} \mathrm{Al}_{2} \mathrm{C}_{0.75}$ (b). Imaginary frequencies are shown as negative values. For $\mathrm{Mo}_{3} \mathrm{Al}_{2} \mathrm{C}$ in panel (a) the calculated phonon dispersion derived from the direct force-constant method (black lines) is compared to results from DFPT theory (red lines). For $\mathrm{Mo}_{3} \mathrm{Al}_{2} \mathrm{C}_{0.75}$ in panel (b) the dispersion relation as calculated from the direct force-constants method (black lines) is presented. It should be noted that because of the reduced symmetry due to the carbon vacancy the dispersions along the paths $\Lambda^{*}= \pm[\xi,-\xi,-\xi]$ and $\Sigma^{*}=\{ \pm[\xi, \xi, 0], \pm[\xi, 0, \xi], \pm[0,-\xi, \xi]\}$ with $0<\xi<\pi / a$ differ to the other symmetry related $\vec{k}$-directions refered by $\Lambda$ and $\Sigma$, respectively.

lizing factor for the vibrational modes. Concomitantly we notice this distortion for the Mo-Al bonding, e.g., the two distinctive nearest Mo-Al bonds with bond-lengths $2.84 \AA$ and $2.95 \AA$ in the fully stoichiometric compound are distorted in $\mathrm{Mo}_{3} \mathrm{Al}_{2} \mathrm{C}_{0.75}$ to lengths in the range of $2.79-3.01 \AA$. Such a distortion for the Mo-Al bonds has recently been indicated experimentally! 10

\section{CARBON VACANCIES}

As discussed, a certain amount of carbon vacancies is needed to stabilize the imaginary optical modes. The key question is if the formation of vacancies is at all thermodynamically possible. This question is investigated by calculating vacancy formation energies and by means of a model.

Within a standard DFT approach, the vacancy formation energy $\varepsilon_{\mathrm{vac}}^{X}$ per atom $X$ is defined by subtracting the total energy $E_{\mathrm{DFT}}\left(\mathrm{Mo}_{12} \mathrm{Al}_{8} \mathrm{C}_{4}\right)$ of the stoichiometric compound from the total energy $E_{\mathrm{DFT}}\left(\mathrm{Mo}_{12} \mathrm{Al}_{8} \mathrm{C}_{4}-X\right)$ of the compound with a vacancy of atom type $X$ and adding the ground-state energy $E_{\mathrm{DFT}}(X)$ of the removed atom $X$ by

$$
\begin{aligned}
\varepsilon_{\mathrm{vac}}^{X}= & E_{\mathrm{DFT}}\left(\mathrm{Mo}_{12} \mathrm{Al}_{8} \mathrm{C}_{4}-X\right)+E_{\mathrm{DFT}}(X)- \\
& -E_{\mathrm{DFT}}\left(\mathrm{Mo}_{12} \mathrm{Al}_{8} \mathrm{C}_{4}\right) .
\end{aligned}
$$

Because standard DFT calculations are strictly valid only at $T=0 \mathrm{~K}$ no temperature dependency has been yet introduced. This can be done by considering the temperature dependent vibrational free energies $F_{\text {vib }}$ and defining the vibrational vacancy formation energy per $X$ atom
TABLE II: Vacancy formation energies in eV for $\mathrm{Mo}_{3} \mathrm{Al}_{2} \mathrm{C}$ for $1 \times 1 \times 1$ and $2 \times 1 \times 1$ supercells without vibrational contributions (DFT, $T=0 \mathrm{~K}$ ) and with $f^{X(\mathrm{vac})}(T)$ at $T=$ $1523 \mathrm{~K}$ and $1773 \mathrm{~K}$.

\begin{tabular}{ccccc}
\hline \hline & Mo-vac & Al-vac & C-vac & C-vac \\
\hline supercell size & $1 \times 1 \times 1$ & $1 \times 1 \times 1$ & $1 \times 1 \times 1$ & $2 \times 1 \times 1$ \\
\hline$\varepsilon$ & 1.74 & 0.86 & 0.60 & 0.56 \\
$T=1523 \mathrm{~K}: \varepsilon+f(T)$ & 1.58 & 0.50 & 0.20 & -0.39 \\
$T=1773 \mathrm{~K}: \varepsilon+f(T)$ & 1.51 & 0.39 & 0.08 & -0.66 \\
\hline C vacancy concentration $x$ for $\mathrm{Mo}_{3} \mathrm{Al}_{2} \mathrm{C}_{1-x}:$ & 0.25 & 0.125 \\
\hline \hline
\end{tabular}

similar to Equ. 1

$$
\begin{aligned}
f_{\mathrm{vac}}^{X}(T)= & F_{\mathrm{vib}}\left(\mathrm{Mo}_{12} \mathrm{Al}_{8} \mathrm{C}_{4}-X\right)+F_{\mathrm{vib}}(X)- \\
& -F_{\mathrm{vib}}\left(\mathrm{Mo}_{12} \mathrm{Al}_{8} \mathrm{C}_{4}\right) .
\end{aligned}
$$

Both, $\varepsilon_{\mathrm{vac}}^{X}$ and $f_{\mathrm{vac}}^{X}(T)$ are formulated for a standard unit cell. To derive results for smaller vacancy concentrations larger supercells are needed and the stoichiometries in Equs. 1 and 2 have to be scaled accordingly. The reference energies $E_{\mathrm{DFT}}(X)$ and $F_{\mathrm{vib}}(X)$ were derived for the ground states of body-centered cubic Mo, face-centered cubic $\mathrm{Al}$, and $\mathrm{C}$ in the graphene structure.

The vacancy formation energies in Table II and Fig. 4 at $T=0 \mathrm{~K}$ are strongly positive for all types of vacancies, whereby the Mo vacancy with its formation energy of $1.74 \mathrm{eV}$ is by far the most unfavorable one. Carbon vacancies are the most favorable ones with a formation energy of $0.60 \mathrm{eV}$ for $\mathrm{Mo}_{3} \mathrm{Al}_{2} \mathrm{C}_{0.75}$. This value is reduced by only $0.04 \mathrm{eV}$ for the smaller vacancy concentration of $\mathrm{Mo}_{3} \mathrm{Al}_{2} \mathrm{C}_{0.875}$.

The experimental samples were synthesized at $T=$ 
(a)

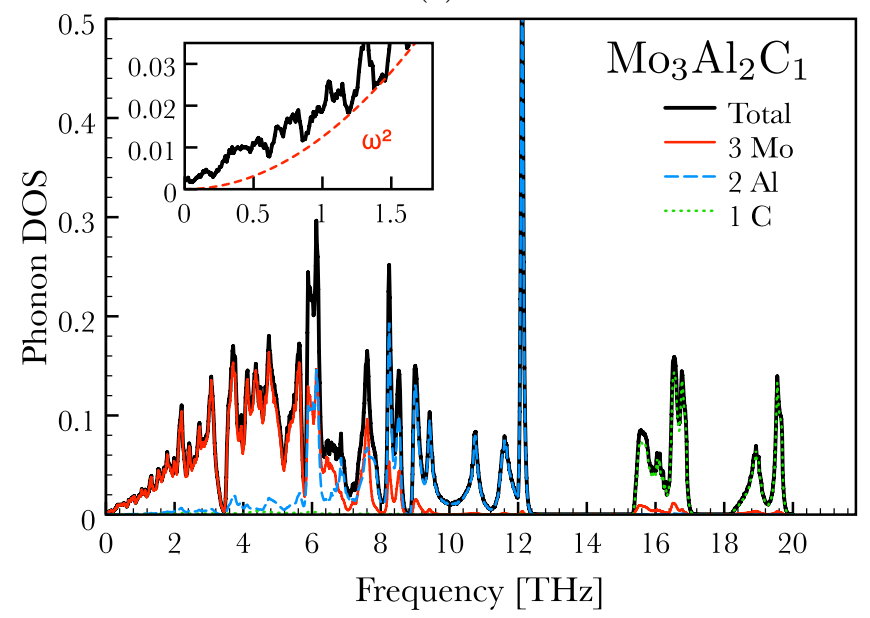

(c)

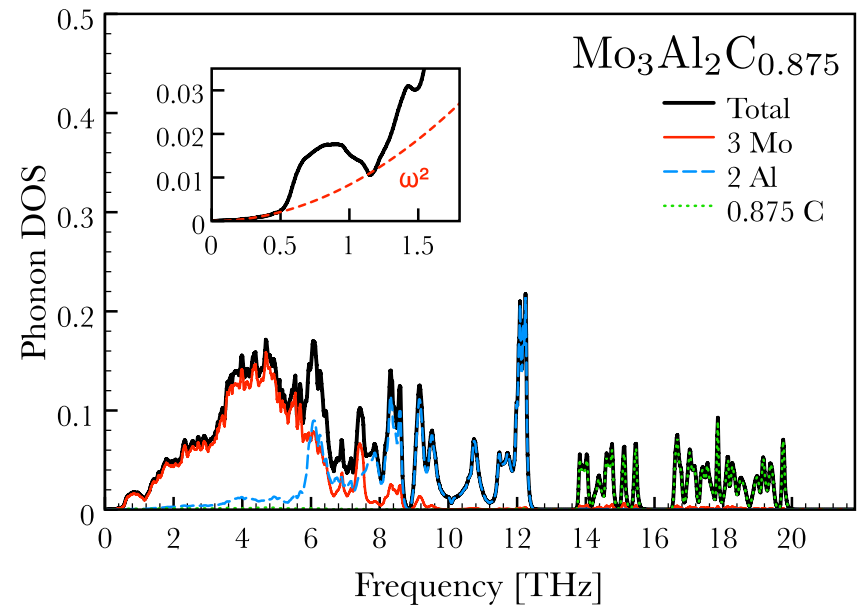

(b)

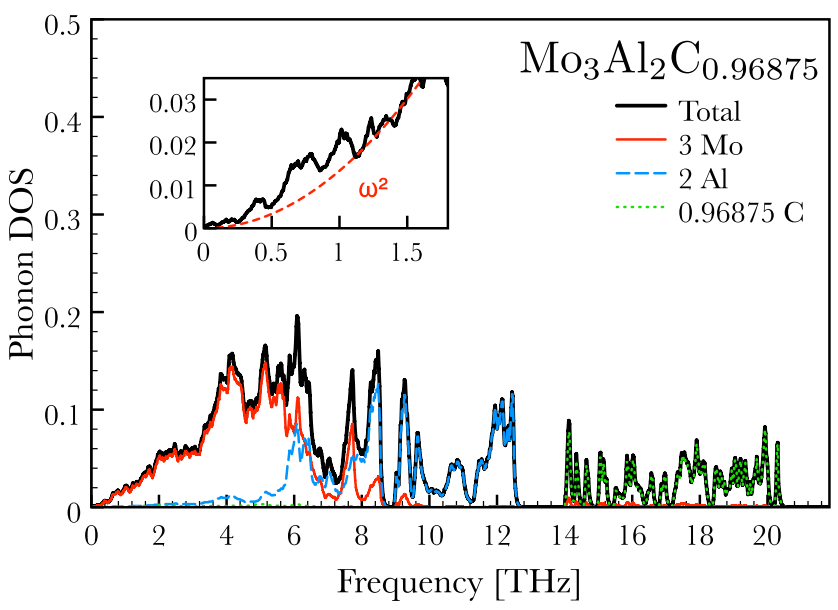

(d)

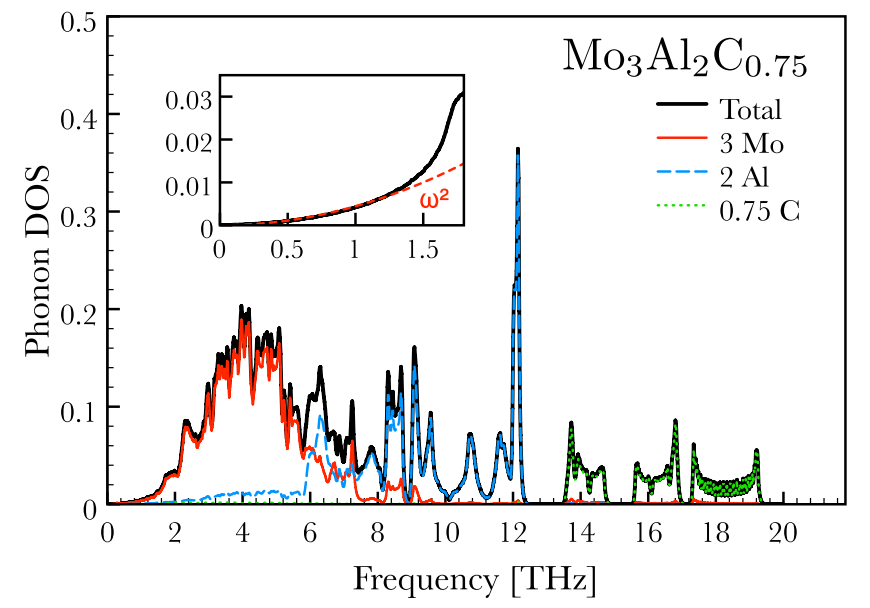

FIG. 2: (Color online) Total and partial phonon DOS of $\mathrm{Mo}_{3} \mathrm{Al}_{2} \mathrm{C}_{1}$ (a), $\mathrm{Mo}_{3} \mathrm{Al}_{2} \mathrm{C}_{0.96875}$ (b), $\mathrm{Mo}_{3} \mathrm{Al}_{2} \mathrm{C}_{0.875}\left(\mathrm{c}\right.$ ), and $\mathrm{Mo}_{3} \mathrm{Al}_{2} \mathrm{C}_{0.75}$ (d) on the Total phonon DOS (black solid line), partial DOS of Mo (red solid line), partial DOS of Al (blue dashed line), and partial DOS of $\mathrm{C}$ (green dotted line). In the insets the total DOS at low frequencies is compared to a Debye-like $\omega^{2}$ behavior (red dashed line).

$1773 \mathrm{~K}$ and heat treated at $1523 \mathrm{~K}]^{6}$ Therefore, theory needs to consider temperature dependent vacancy formation energies $f_{\text {vac }}^{X}(T)$ combined with the composition dependent configurational entropy $S_{\text {conf }}(x)^{\sqrt{24}}$ in order to compare with experiment. For the actual calculation of the vibrational free energy the small amount of imaginary modes in the fully stoichiometric compound $\mathrm{Mo}_{3} \mathrm{Al}_{2} \mathrm{C}_{1}$ were omitted.

Table $\mathrm{II}$ and Fig. 4 show that the vibrational contributions reduce the strongly positive vacancy formation energies at $T=0 \mathrm{~K}$. While this reduction is rather small for the Mo vacancy (from $1.74 \mathrm{eV}$ to $1.51 \mathrm{eV}$ at $1773 \mathrm{~K}$ ), it is much larger for the other two types of vacancies.

In particular, the formation energy of the carbon vacancy in $\mathrm{Mo}_{3} \mathrm{Al}_{2} \mathrm{C}_{0.75}$ decreases from $0.60 \mathrm{eV}$ to $0.08 \mathrm{eV}$ at $1773 \mathrm{~K}$. Remarkably, this reduction is much larger for the smaller carbon vacancy concentration, i.e., $\mathrm{Mo}_{3} \mathrm{Al}_{2} \mathrm{C}_{0.875}$, with a decrease by more than $1 \mathrm{eV}$ down to $-0.66 \mathrm{eV}$. These negative values for the formation energy indicate a possible thermodynamical stabilization of carbon vacancies in $\mathrm{Mo}_{3} \mathrm{Al}_{2} \mathrm{C}_{1-x}$. Noticeably, this difference of the temperature dependent free energies for different carbon vacancy concentrations comes exclusively from the vibrational contributions $f(x, T)$, because -as mentioned above- at $T=0 \mathrm{~K}$ the vacancy formation energies are almost equal.

From an isolated defect model ${ }^{25}$ the temperature dependent equilibrium vacancy concentration $x$ can be calculated. However, the vacancy formation energy $\varepsilon(x)+f(x, T)$ in the description of the internal energy, $U(x, T)=(\varepsilon(x)+f(x, T)) x, \underline{26}$ is strongly dependent on $x$. Hence, the isolated defect model cannot be applied 


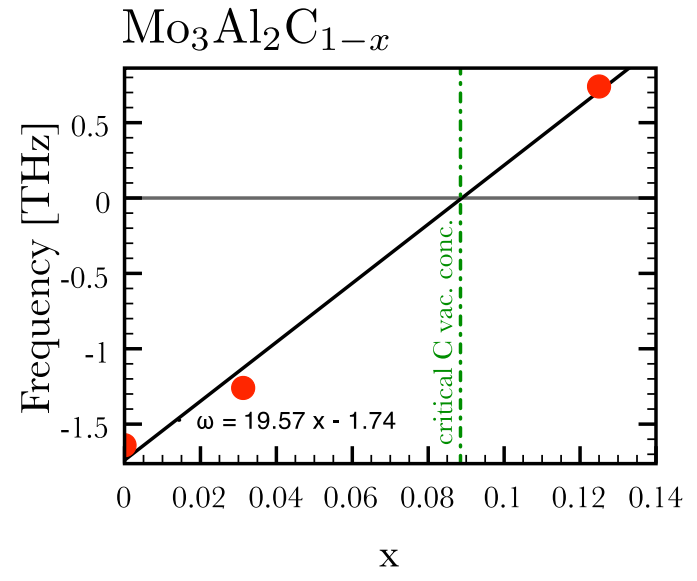

FIG. 3: (Color online) Frequency of the lowest optical mode at $\Gamma$ versus carbon vacancy concentration $x$ as calculated (red circles) and linearly interpolated (solid black line). The predicted critical carbon vacancy concentration is indicated (green dashed-dotted line).

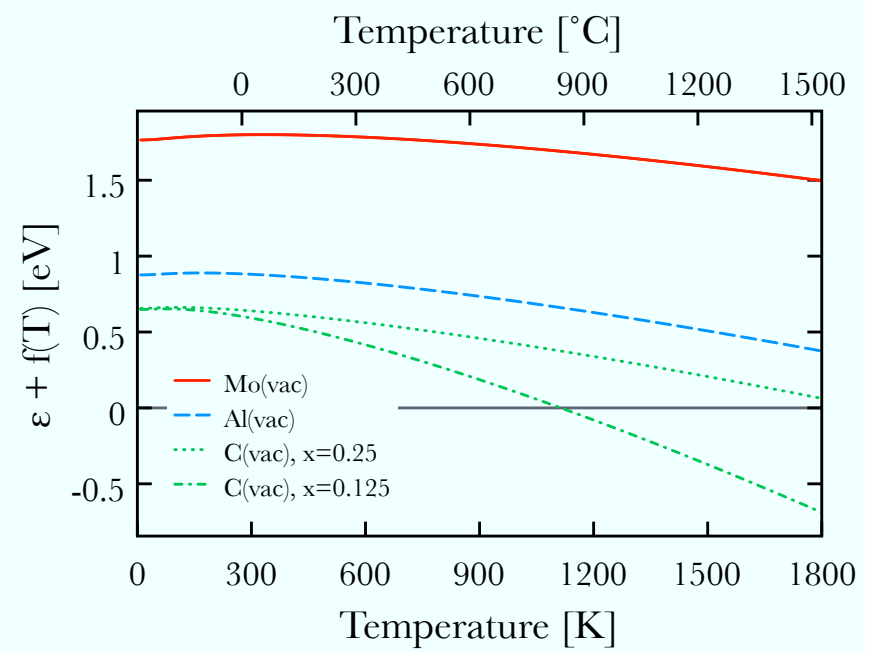

FIG. 4: (Color online) Vacancy formation energy $\varepsilon+f(T)$ as the sum of the DFT $\varepsilon$ and vibrational $f(T)$ formation energy versus temperature for a Mo vacancy (red solid line), a $\mathrm{Al}$ vacancy (blue dashed line), a $\mathrm{C}$ vacancy (green dotted line) in a unit cell $(1 \times 1 \times 1$ supercell), and for a $C$ vacancy in a $2 \times 1 \times 1$ supercell (green dashed-dotted line).

directly, as the internal energy $U(x, T)$ is not a linear function of $x$. In our case it is described as a quadratic function of $x, U(x, T)=a x^{2}+b x$ wherein $a$ and $b$ are temperature-dependent parameters fitted to the calculated carbon values at $x=0,0.125$, and 0.25 .

Thus, the free energy for the vacancy formation including the configurational entropy is formulated as

$$
F(x, T)=a x^{2}+b x-k_{B} T S_{\text {conf }}(x) .
$$

Assuming $S_{\text {conf }}(x)$ is the mixing entropy of non-

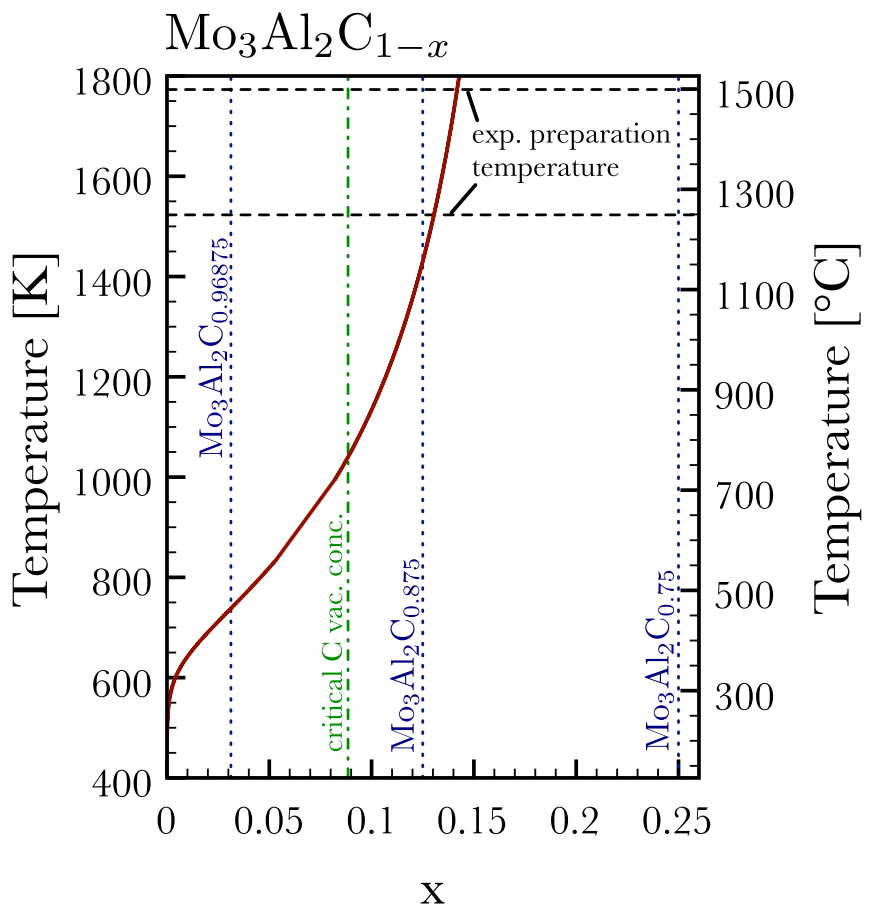

FIG. 5: (Color online) Temperature dependent $\mathrm{C}$ vacancy concentration $x(T)$ plotted as a solid red line. Experimental preparation temperatures are indicated as dashed black lines. The critical $\mathrm{C}$ vacancy concentration, below which $\mathrm{Mo}_{3} \mathrm{Al}_{2} \mathrm{C}_{1-x}$ gets dynamically unstable, is shown as a green dash-dotted line, while the $\mathrm{C}$ vacancy concentrations of the calculated supercells are drawn as blue dotted lines.

interacting vacancies $S_{\text {conf }}(x)=x \ln (x)+(1-x) \ln (1-x)$, the derivative of $F$ with respect to $x$ can be used to search for the temperature dependent concentration $x(T)$ by minimizing the free energy, i.e.,

$$
\frac{\partial F(x, T)}{\partial x}=0 \quad \Rightarrow \quad x=\frac{e^{-\beta(2 a x+b)}}{1+e^{-\beta(2 a x+b)}},
$$

with $\beta=1 /\left(k_{B} T\right)$. This expression enables the numerical calculation of $x(T)$, as shown in Fig. 5, using a bracketing root finding algorithm.27 Inspecting Fig. 5 the C vacancy concentration is $0.13-0.14$. at the experimental preparation temperatures ${ }^{6}$ of $1773 \mathrm{~K}$ and $1523 \mathrm{~K}$. As elaborated in the previous Section III at such vacancy concentrations $\mathrm{Mo}_{3} \mathrm{Al}_{2} \mathrm{C}_{1-x}$ is dynamically stable.

\section{ELECTRONIC STRUCTURE}

After finding that vacancies do exist on the carbon sublattice and that these are necessary to stabilize the crystal structure we will now briefly discuss the band structure and the electronic DOS of $\mathrm{Mo}_{3} \mathrm{Al}_{2} \mathrm{C}_{1-x}$. As will be shown the influence of a changed carbon stoichiometry on the band structure can not be described by a simple rigid 
(a)

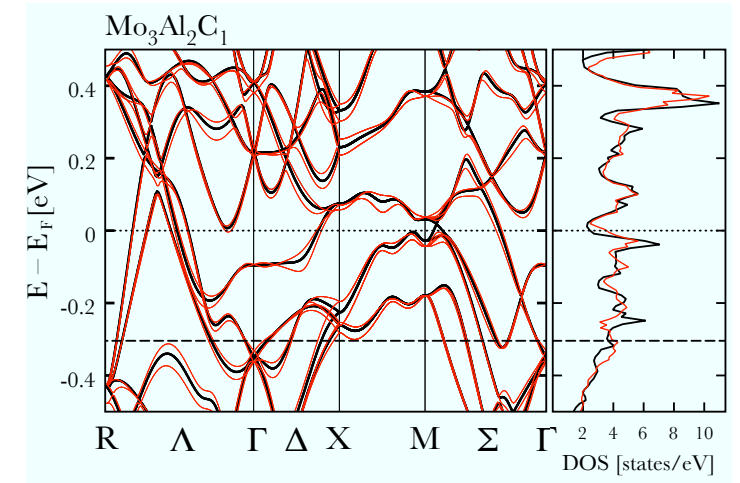

(b)

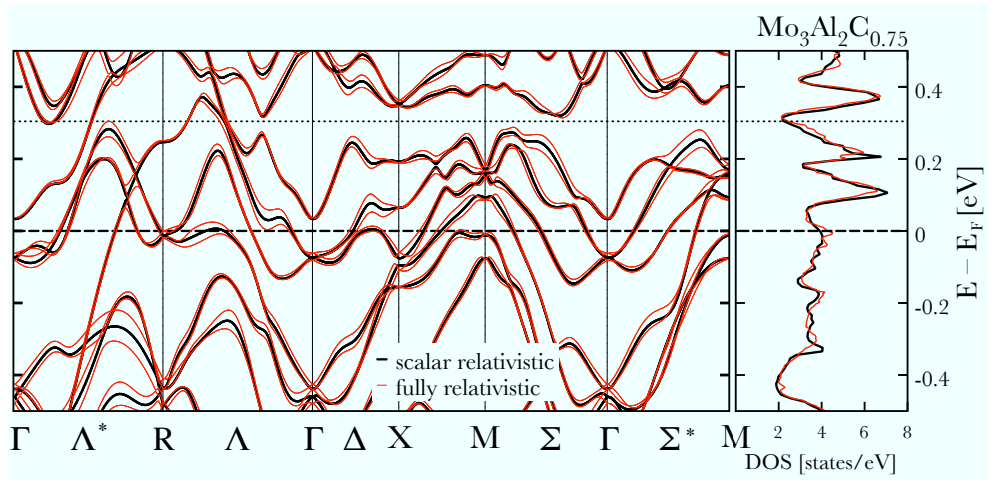

FIG. 6: (Color online) Electronic band structure and DOS of stoichiometric $\mathrm{Mo}_{3} \mathrm{Al}_{2} \mathrm{C}$ (a) and of $\mathrm{Mo}_{3} \mathrm{Al}_{2} \mathrm{C}_{0.75}$ (b) calculated scalar relativistically (black lines) and fully relativistically including spin-orbit coupling (red lines). The Fermi energy $E_{\mathrm{F}}$ referring to the number of valence electrons of $\mathrm{Mo}_{3} \mathrm{Al}_{2} \mathrm{C}$ is indicated as a dotted line while the Fermi energy corresponding to $\mathrm{Mo}_{3} \mathrm{Al}_{2} \mathrm{C}_{0.75}$ is indicated as a dashed line. Note the different directions $\Sigma, \Sigma^{*}$ and $\Lambda, \Lambda^{*}$ as discussed in the caption of Fig. 1 .

band model. Especially, the spin-orbit splitting on the bands in a fully relativistic calculation strongly depends on $x$.

In Fig. 6 the electronic band structure and DOS of the fully stoichiometric compound is compared to that of $\mathrm{Mo}_{3} \mathrm{Al}_{2} \mathrm{C}_{0.75}$. The attentive reader might question this choice of $x=0.25$ as being too high, because our thermodynamical model (described in the previous section) predicted a much lower carbon vacancy concentration. However, we chose this value due to the fact that the unit cells of both compounds have equal size and shape making it easier to compare these.

At this point it should be remarked that our calculated band structure for $\mathrm{Mo}_{3} \mathrm{Al}_{2} \mathrm{C}_{1}$ shown in panel (a) in Fig. 6 resembles the one published previously by Bauer et $a l]^{6}$ but differs distinctively from that published by Karki et $a l^{17}$ The different finding by Karki et al. can only stem from the use of a different crystal structure. We have recalculated the band structure by means of the fullpotential linearized augmented plane-wave method using our own code FLAIR ${ }^{28 / 29}$ and also by comparing to a recent calculation with the code Wien $2 \mathrm{~K}^{[30}$ (the later was also used by Karki et al ${ }^{\text {[7)}}$. All these calculations yielded the same result for the band structure of $\mathrm{Mo}_{3} \mathrm{Al}_{2} \mathrm{C}_{1}$ in the cubic $\beta$-Mn type crystal structure, i.e., the one shown in panel (a) of Fig. 6.

We have calculated the band structures both in a scalar relativistic approximation, omitting spin-orbit coupling, and fully relativistically, including spin-orbit coupling in a self-consistent manner. As expected, some degeneracies at the high-symmetry points are different whether spin-orbit coupling is included or not, e.g., a splitting of $30 \mathrm{meV}$ at $\Gamma$ and of $53 \mathrm{meV}$ at $\mathrm{R}$ in $\mathrm{Mo}_{3} \mathrm{Al}_{2} \mathrm{C}_{1}$ and of $20 \mathrm{meV}$ at both $\Gamma$ and $\mathrm{R}$ in $\mathrm{Mo}_{3} \mathrm{Al}_{2} \mathrm{C}_{0.75}$ just below the Fermi energy $E_{\mathrm{F}}$ is evident. However, the most striking point is the loss of the double degeneracy of the bands due to spin-orbit coupling in noncentrosymmetric compounds ${ }^{3132}$ From Fig. 6 we observe this vertical spin-orbit splitting of the bands, e.g., $65 \mathrm{meV}$ on the path $\Lambda$ around $-0.35 \mathrm{eV}$ in $\mathrm{Mo}_{3} \mathrm{Al}_{2} \mathrm{C}_{1}$ and $90 \mathrm{meV}$ on the path $\Lambda^{*}$ around $-0.3 \mathrm{eV}$ in $\mathrm{Mo}_{3} \mathrm{Al}_{2} \mathrm{C}_{0.75}$. As a consequence the Fermi surfaces of noncentrosymmetric $\mathrm{Mo}_{3} \mathrm{Al}_{2} \mathrm{C}_{1-x}$ do also split due to spin-orbit coupling which can be seen in Fig. 6] as the horizontal band splitting at $E_{\mathrm{F}}$.

Comparing the band structure and electronic DOS of $\mathrm{Mo}_{3} \mathrm{Al}_{2} \mathrm{C}_{1}$ with that of $\mathrm{Mo}_{3} \mathrm{Al}_{2} \mathrm{C}_{0.75}$ in Fig. 6 one immediately notices that removing a carbon atom from the carbon sublattice has a substantial effect and it is not sufficient to simply shift the Fermi level in order to account for the carbon vacancy.

From these results we conclude, that the structure of the Fermi surfaces and hence any nesting, paramount to the understanding of superconductivity, strongly depend on $x$.

\section{CONCLUSIONS}

In the present work we have shown, that vacancies are necessary to dynamically stabilize the cubic $\beta$-Mn type $\left(\mathrm{P} 4_{1} 32\right)$ crystal structure of $\mathrm{Mo}_{3} \mathrm{Al}_{2} \mathrm{C}$, whereby vacancies on the carbon sublattice are energetically the most favorable ones. According to our thermodynamical model the most probable carbon vacancy concentration $x$ in $\mathrm{Mo}_{3} \mathrm{Al}_{2} \mathrm{C}_{1-x}$ is about $0.13-0.14$ considering actual experimental preparation temperatures.

We have demonstrated that there exists a critical value of $x_{\text {crit }} \sim 0.09$ below which $\mathrm{Mo}_{3} \mathrm{Al}_{2} \mathrm{C}_{1-x}$ becomes dynamically unstable, and especially the frequency at which the Debye-like behavior of the phonons ends strongly depends on $x$.

Likewise, the band structure and electronic DOS are influenced by the carbon vacancy concentration.

The still unresolved question if $\mathrm{Mo}_{3} \mathrm{Al}_{2} \mathrm{C}$ is a conventional or unconventional superconductor can only be an- 
swered when the carbon vacancies are properly considered, as the structure and nesting of the Fermi surfaces depend on the carbon vacancy concentration. If this carbon vacancy concentration could be controlled, it might be possible to tune the superconducting properties of $\mathrm{Mo}_{3} \mathrm{Al}_{2} \mathrm{C}_{1-x}$. This might be rather difficult, because it may depend on the sample preparation and the cooling process. Further, one can safely assume, that at the preparation temperature of $\approx 1500 \mathrm{~K}$ the sample is in its thermodynamic equilibrium. This is not the case when its superconducting properties are measured where it is in a quenched meta-stable state.

\section{Acknowledgments}

This work was supported by the Austrian Science Fund FWF under Grant No. P22295. Computational calculations were done on the Vienna Scientific Cluster (VSC).
* Electronic address: david.reith@univie.ac.at

1 W. Jeitschko, H. Nowotny, and F. Benesovsky, Monatshefte für Chemie / Chemical Monthly 94, 247 (1963).

2 J. Johnston, L. Toth, K. Kennedy, and E. R. Parker, Solid State Commun. 2, 123 (1964).

3 H. J. Fink, A. C. Thorsen, E. Parker, V. F. Zackay, and L. Toth, Phys. Rev. 138, A1170 (1965).

${ }^{4}$ L. Toth and J. Zbasnik, Acta Metall. 16, 1177 (1968).

${ }^{5}$ L. E. Toth, Transition Metal Carbides and Nitrides. Refractory Materials Monographs., vol. 7 (Academic Press, New York, 1971).

${ }^{6}$ E. Bauer, G. Rogl, X.-Q. Chen, R. T. Khan, H. Michor, G. Hilscher, E. Royanian, K. Kumagai, D. Z. Li, Y. Y. Li, et al., Phys. Rev. B 82, 064511 (2010).

7 A. B. Karki, Y. M. Xiong, I. Vekhter, D. Browne, P. W. Adams, D. P. Young, K. R. Thomas, J. Y. Chan, H. Kim, and R. Prozorov, Phys. Rev. B 82, 064512 (2010).

8 I. Bonalde, H. Kim, R. Prozorov, C. Rojas, P. Rogl, and E. Bauer, Phys. Rev. B 84, 134506 (2011).

9 T. Koyama, Y. Ozaki, K. Ueda, T. Mito, T. Kohara, T. Waki, Y. Tabata, C. Michioka, K. Yoshimura, M.-T. Suzuki, et al., Phys. Rev. B 84, 212501 (2011).

10 C. N. Kuo, H. F. Liu, and C. S. Lue, Phys. Rev. B 85, 052501 (2012).

11 E. Bauer, G. Hilscher, H. Michor, C. Paul, E. W. Scheidt, A. Gribanov, Y. Seropegin, H. Noël, M. Sigrist, and P. Rogl, Phys. Rev. Lett. 92, 027003 (2004).

12 L. P. Gor'kov and E. I. Rashba, Phys. Rev. Lett. 87, 037004 (2001).

13 M. Sigrist, D. Agterberg, P. Frigeri, N. Hayashi, R. Kaur, A. Koga, I. Milat, and K. W. Y. Yanase, J. Magn. Magn. Mater. 310, 536 (2007).

14 G. Kresse and J. Furthmüller, Phys. Rev. B 54, 11169 (1996).

15 G. Kresse and D. Joubert, Phys. Rev. B 59, 1758 (1999).
16 P. E. Blöchl, Phys. Rev. B 50, 17953 (1994).

17 J. P. Perdew, K. Burke, and M. Ernzerhof, Phys. Rev. Lett. 77, 3865 (1996).

18 P. E. Blöchl, O. Jepsen, and O. K. Andersen, Phys. Rev. B 49, 16223 (1994).

19 H. Monkhorst and J. Pack, Phys. Rev. B 13, 5188 (1976).

20 M. Methfessel and A. T. Paxton, Phys. Rev. B 40, 3616 (1989).

21 D. Alfè, Comp. Phys. Commun. 180, 2622 (2009).

22 S. Baroni, S. de Gironcoli, A. Dal Corso, and P. Giannozzi, Rev. Mod. Phys. 73, 515 (2001).

${ }^{23}$ W. H. McMaster, N. D. Grande, J. Mallett, and J. Hubbell, Compilation of X-Ray Cross Sections (Lawrence Livermore National Laboratory Report UCRL-50174 Section II Revision I, 1969).

24 D. Reith and R. Podloucky, Physical Review B 80, 054108 (2009).

25 N. W. Ashcroft and N. D. Mermin, Festkörperphyik (Oldenbourg Verlag München Wien, 2001), chap. 30, pp. 782785, translation by J. Gress.

26 J. Mayer and M. Fähnle, Acta Materialia 45, 2207 (1997).

27 W. H. Press, B. P. Flannery, S. A. Teukolsky, and W. T. Vetterling, Numerical Recipes: The Art of Scientific Computing (Cambridge University Press, 2007), p. 445.

28 M. Weinert, E. Wimmer, and A. J. Freeman, Phys. Rev. B 26, 4571 (1982).

29 M. Weinert, G. Schneider, R. Podloucky, and J. Redinger, J. Phys.: Condens. Matter 21, 084201 (2009).

30 P. Blaha (2012), private communication.

31 J. Callaway, Energy Band Theory (Academic Press, New York and London, 1964), chap. 1.

32 E. Bauer, R. T. Khan, H. Michor, E. Royanian, A. Grytsiv, N. Melnychenko-Koblyuk, P. Rogl, D. Reith, R. Podloucky, E.-W. Scheidt, et al., Phys. Rev. B 80, 064504 (2009). 